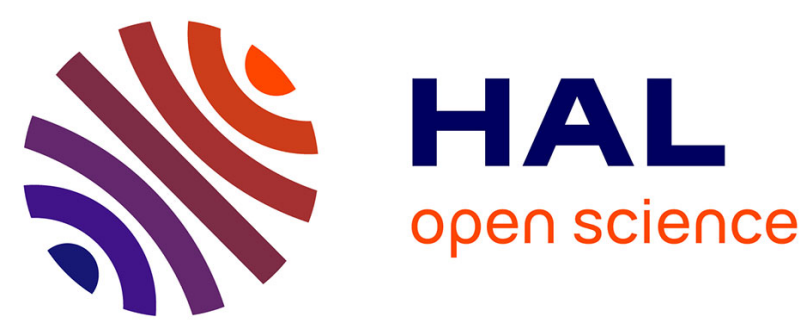

\title{
Sparse reconstruction methods in x-ray CT
}

Juan F P J Abascal, Monica Abella, Cyril Mory, Nicolas Ducros, Claudia de Molina, Eugenio Marinetto, Françoise Peyrin, Manuel Desco

\section{To cite this version:}

Juan F P J Abascal, Monica Abella, Cyril Mory, Nicolas Ducros, Claudia de Molina, et al.. Sparse reconstruction methods in X-ray CT. Developments in X-Ray Tomography XI, Aug 2017, San Diego, United States. 10.1117/12.2272711 . hal-01737088

\section{HAL Id: hal-01737088 \\ https://hal.science/hal-01737088}

Submitted on 19 Mar 2018

HAL is a multi-disciplinary open access archive for the deposit and dissemination of scientific research documents, whether they are published or not. The documents may come from teaching and research institutions in France or abroad, or from public or private research centers.
L'archive ouverte pluridisciplinaire HAL, est destinée au dépôt et à la diffusion de documents scientifiques de niveau recherche, publiés ou non, émanant des établissements d'enseignement et de recherche français ou étrangers, des laboratoires publics ou privés. 


\title{
Sparse reconstruction methods in X-ray CT
}

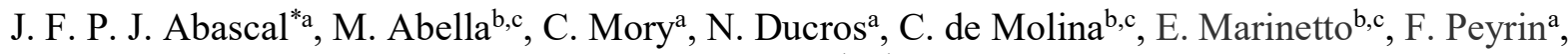 \\ M. Desco ${ }^{\text {b,c,d }}$ \\ aUniv.Lyon, INSA-Lyon, Université Claude Bernard Lyon 1, UJM-Saint Etienne, CNRS, Inserm, \\ CREATIS UMR 5220, U1206, F-69100, Lyon, France;

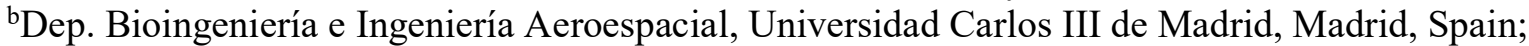 \\ 'Instituto de Investigación Sanitaria Gregorio Marañón (IiSGM), Madrid, Spain; \\ ${ }^{\mathrm{d} C e n t r o ~ d e ~ I n v e s t i g a c i o ́ n ~ e n ~ R e d ~ d e ~ S a l u d ~ M e n t a l ~(C I B E R S A M), ~ M a d r i d, ~ S p a i n ~}$
}

\begin{abstract}
Recent progress in X-ray CT is contributing to the advent of new clinical applications. A common challenge for these applications is the need for new image reconstruction methods that meet tight constraints in radiation dose and geometrical limitations in the acquisition. The recent developments in sparse reconstruction methods provide a framework that permits obtaining good quality images from drastically reduced signal-to-noise-ratio and limited-view data.

In this work, we present our contributions in this field. For dynamic studies (3D+Time), we explored the possibility of extending the exploitation of sparsity to the temporal dimension: a temporal operator based on modelling motion between consecutive temporal points in gated-CT and based on experimental time curves in contrast-enhanced CT. In these cases, we also exploited sparsity by using a prior image estimated from the complete acquired dataset and assessed the effect on image quality of using different sparsity operators. For limited-view CT, we evaluated total-variation regularization in different simulated limited-data scenarios from a real small animal acquisition with a cone-beam microCT scanner, considering different angular span and number of projections. For other emerging imaging modalities, such as spectral CT, the image reconstruction problem is nonlinear, so we explored new efficient approaches to exploit sparsity for multi-energy CT data.

In conclusion, we review our approaches to challenging $\mathrm{CT}$ data reconstruction problems and show results that support the feasibility for new clinical applications.
\end{abstract}

Keywords: Sparsity, compressed sensing, L1-norm, split Bregman, contrast-enhanced CT, respiratory gated-CT, limited view CT, spectral CT

\section{INTRODUCTION}

Recent progress in X-ray CT is contributing to the advent of new clinical applications. A common challenge for these applications is the need for new image reconstruction methods that meet tight constraints in radiation dose and geometrical limitations in the acquisition.

The revolutionary discovery of compressed sensing (CS) provides a framework that enables accurate image reconstruction from few noisy projections using convex optimization, provided that the image is sufficiently sparse in a transformed domain and that uniform random sampling and an incoherence condition hold. ${ }^{1}$ Given the success of CS, sparse regularization has become a preferred choice for designing iterative regularized schemes even if the CS assumptions are not met. Recent theoretical work also aims to extend the CS framework to more practical measurement conditions and for nonlinear measurement operators. ${ }^{2,3}$ Specifically, in x-ray imaging, sparsity has been proposed to improve image quality in cases of reduced signal-to-noise-ratio and limited-view data., ${ }^{4,5}$

In this work, we present our contributions to exploiting sparsity for solving challenging problems in X-ray CT. Most of our contributions focus on high dimensionality problems, for which data redundancy and image sparsity can be highly

* Juan.abascal@,creatis.insa-lyon.fr 
exploited. For dynamic studies (3D+Time), we have explored the possibility of extending the exploitation of sparsity to the temporal dimension. In this case, the Prior Image Constrained Compressed Sensing (PICCS) algorithm leads to superior results than the widely used total variation method, as images are highly sparse when subtracted from a prior image obtained by combining all data. ${ }^{6-8}$

In respiratory gated-CT, we have explored several methods. We assessed the effect on image quality of using different sparsity operators within PICCS algorithm. An improved method obtained a sparser temporal representation by modelling motion between consecutive temporal points. We proposed two different motion-based reconstruction approaches. PRIMOR method modelled motion using B-splines and solved the optimization problem using the Split Bregman formulation. ${ }^{9}$ The second approach, named MA-ROOSTER, was applied to free breathing respiratory cone beam CT data. The optimization framework is empirical: it alternates between iterations of conjugate gradient to minimize a 4D data-attachment term, and several denoising steps: enforcing non-negativity, canceling motion outside the patient, spatial 3D TV, and temporal 1D TV along curved trajectories determined by a priori motion. ${ }^{10}$ The need for a reasonable a priori motion, e.g. estimated from a previously existing $4 \mathrm{D}$ reconstruction, is critical for MA-ROOSTER. In radiation therapy, its main target, patients undergo a 4D CT before treatment, therefore the prior information on motion is available in clinical routine.

In contrast-enhanced $\mathrm{CT}$, we proposed an alternative formulation to regularization based on replacing the original problem by a new one that is well conditioned. This approach was specifically designed for the case of slow rotating scanners for preclinical imaging. The idea was inspired on previous work where the solution was a combination of few temporal basis functions that modelled the contrast uptake. ${ }^{11}$ We reformulated the reconstruction problem as the recovery of piecewise cubic polynomials in the temporal dimension, which significantly reduced the number of degrees of freedom. ${ }^{12}$

In limited-view CT, where only a few projections are taken in a reduced angular span, analytical reconstruction methods provide low quality images with lots of artifacts. We evaluated the viability of total variation method to solve this problem using numerical simulations based on geometrical description of a real scanner. ${ }^{13,14}$

For other emerging imaging modalities, such as spectral $\mathrm{CT}$, the image reconstruction problem is commonly divided in two steps, the material decomposition problem, which is nonlinear, and the tomographic step. Mostly all studies that proposed regularized- or sparsity-based approaches focused on the tomographic step. The material decomposition step is nonlinear and so it is more challenging to solve. In this problem, we have recently proposed an efficient regularized Gauss Newton approach to exploit specific regularization for each material. ${ }^{15}$

\section{RESPIRATORY GATED CT}

Respiratory gating helps to overcome the problem of breathing motion in cardiothoracic imaging, which is relevant in several clinical situations. ${ }^{16,17}$ The gating process assigns acquired projections to the different respiratory phases, which leads to insufficient data for the reconstruction of each phase when using standard reconstruction algorithms. Hence, using conventional reconstruction methods requires acquiring more data, which results in a substantial increase in the radiation dose. In this section, we propose several sparse reconstruction methods that exploit sparsity in the temporal dimension.

\subsection{Investigation of different sparsity transforms for PICCS algorithm}

Prior image constrained compressed sensing (PICCS) has been proposed for reconstructing gated CT data from highly undersampled and noisy data. ${ }^{7,8}$ PICCS has led to significant improvements in image quality with respect to the total variation method, in a wide variety of applications. Its success is because reconstructed images are highly sparse when subtracted from a prior image, which is obtained by combining all data. PICCS solves the convex constrained optimization problem

$$
\min _{u}(1-\alpha)\|\Psi(u)\|_{1}+\alpha\left\|\Phi\left(u-u_{p}\right)\right\|_{1} \quad \text { st. } \quad\|R u-p\|_{2}^{2} \leq \sigma^{2}
$$

where $u$ represents the reconstructed gates, $u_{p}$ represent the prior image, $p$ represents the raw data for all respiratory phases, $R$ represents the forward operator, $\sigma^{2}$ accounts for noise in the data, and $\alpha$ weights the image prior penalty function. The first and second terms in (1) impose spatial sparsity and temporal sparsity with respect to the prior image, 
respectively. The common choice for the transformations $\Psi$ and $\Phi$ is the spatial discrete gradient that leads to total variation functional. However, other functional may lead to superior results.

In this work, we evaluated the suitability of different sparsity transforms for $\Phi$ (unitary, gradient, and symlet wavelet transform) within the PICCS formulation for reducing dose in CT respiratory gating for small-animal imaging. ${ }^{18}$ This lead to three different flavors of PICCS: L1-PICCS, TV-PICS and WT-PICCS. Methods were assessed in different scenarios, corresponding to different X-ray flux levels and number of projections. Problem (1) was solved using the Split Bregman formulation, which efficiently solves L1-regularized problems. ${ }^{19,20}$ All flavors of PICCS performed very similarly in terms of noise, spatiotemporal resolution, and streak reduction. Nevertheless, the wavelet transform led to superior image texture than the other transforms (figure 1). The gradient and unitary transforms led to staircase-like artefacts and pixel-like artefacts, respectively. PICCS led to significantly improvement in image quality with respect to filtered back-projection and allowed to reduce the respiratory motion artefact.

\section{L1-PICCS}

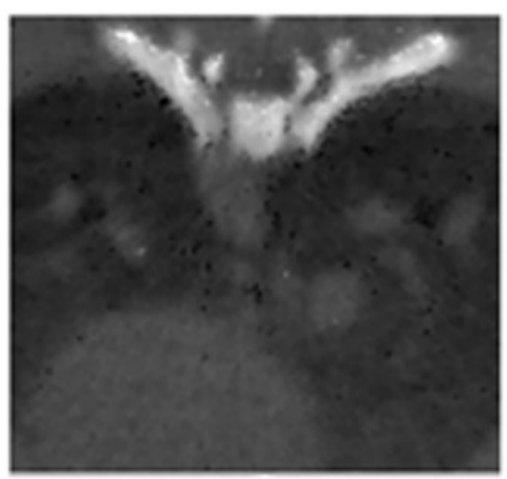

TV-PICCS

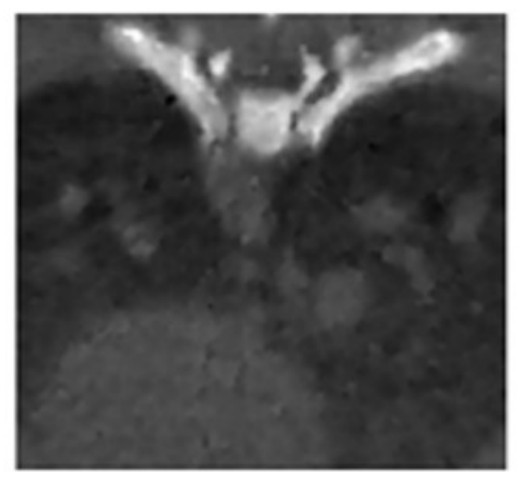

\section{WT-PICCS}

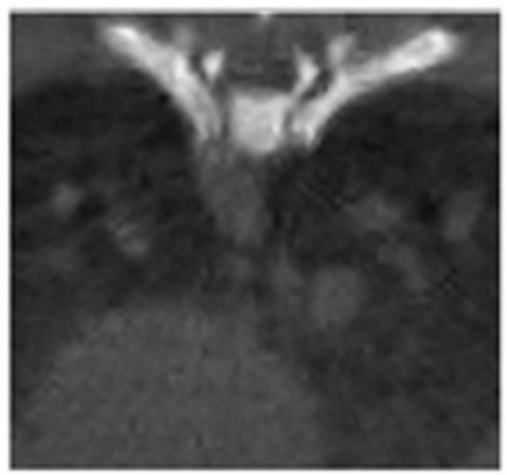

Figure 1. Zoomed-in images of reconstructed images with the different methods for one respiratory phase. Gated data consisted of 120 projections per respiratory phase and X-ray flux corresponding to a number of photons $\mathrm{I}_{0}=4.5 \cdot 10^{4}$.

\subsection{PRIMOR - A prior- and motion-based reconstruction method}

In this work, we proposed a prior- and motion-based compressed sensing (PRIMOR) method for respiratory gating in small-animal CT. ${ }^{21}$ PRIMOR is a combination of both prior- and motion-based reconstruction methods. Thus, it benefits from the advantages of these two approaches. On the one hand, it exploits the available prior image, and on the other hand, it takes into account motion between consecutive respiratory gates, which leads to sparser representation in the temporal dimension.

PRIMOR is implemented as a two-step approach. In the first step, motion is estimated using a nonrigid registration method based on hierarchical B-splines. ${ }^{22}$ In the second step, only image variation with respect to the prior is reconstructed using the previously estimated motion. PRIMOR method solved the problem

$$
\min _{v} \beta\left\|\Psi\left(u_{p}+v\right)\right\|_{1}+\alpha\|\Phi v\|_{1}+\gamma\left\|\mathrm{T}\left(u_{p}+v\right)\right\|_{1} \quad \text { st. } \quad\left\|R\left(u_{p}+v\right)-p\right\|_{2}^{2} \leq \sigma^{2}
$$

where $u_{p}$ is the prior image, $v$ is the image variation with respect to the prior image, $u=u_{p}+v$, and $T$ is an operator that encodes motion between respiratory phases. PRIMOR was solved using the split Bregman formulation and was compared to an equivalent prior-based method without motion estimation on different simulated scenarios (MATLAB code for PRIMOR method can be found https://github.com/HGGM-LIM/prior-motion-reconstruction-CT). Both PRIMOR and prior-based methods greatly improved FDK reconstruction in all scenarios. The prior-based method was prone to streak artefacts and noise when using a low number of projections or low dose. PRIMOR corrected for these effects, leading to better contrast recovery, less error and improved motion artefact correction (figure 2). 


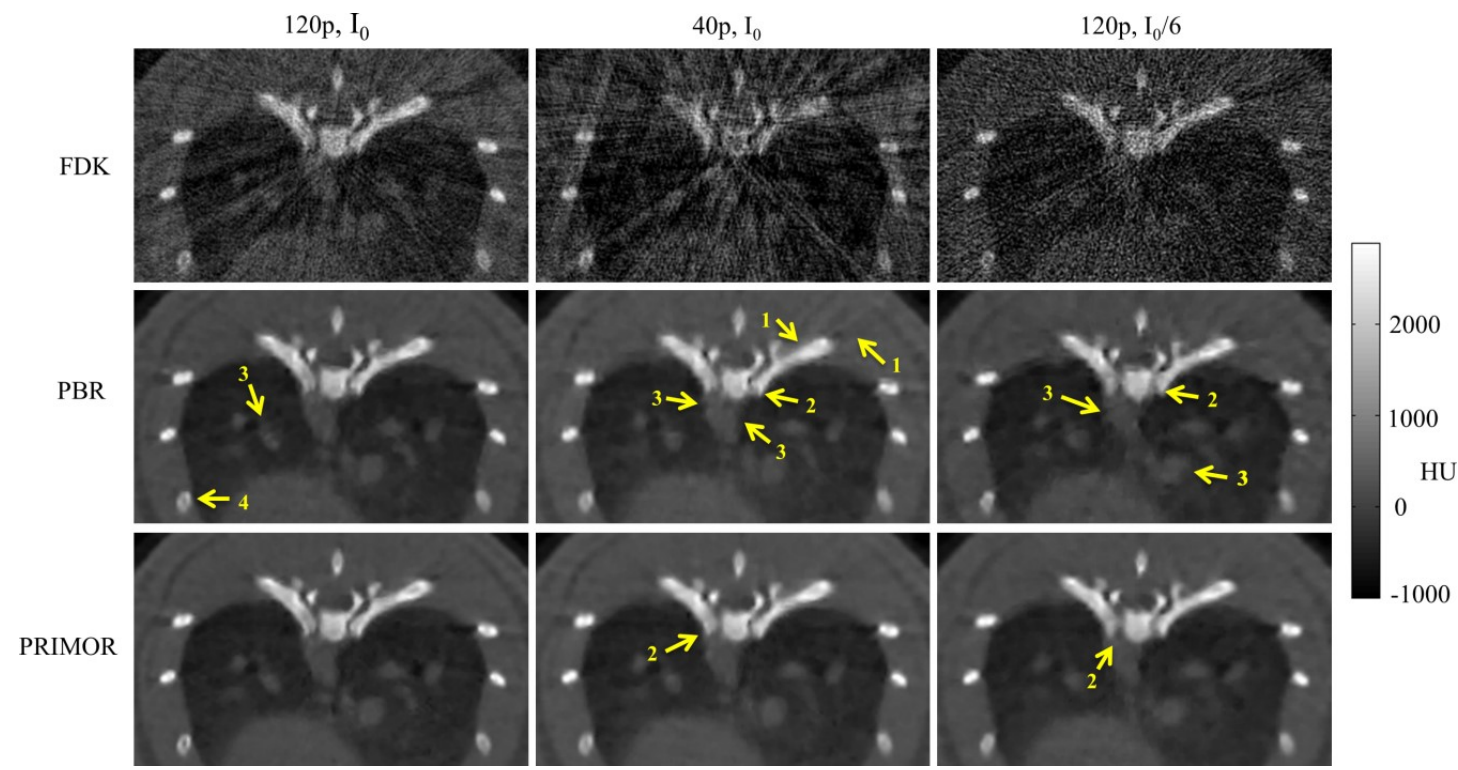

Figure 2. Zoomed-in of reconstructed images with FDK, prior-based method (PBR) and PRIMOR method, for different scenarios corresponding to different number of projections and number of photons $\left(\mathrm{I}_{0}=4.5 \cdot 10^{4}\right)$. Yellow arrows indicate where artifacts are more noticeable: an increase in streak artifacts (1), blurred edges for bone tissue (2), blurred edges for soft tissue (3), loss of contrast resolution within bone tissue (4).

\subsection{MA-ROOSTER - Motion-Aware RecOnstructiOn using Spatial and TEmporal Regularization}

MA-ROOSTER assumes that a motion mask, i.e. a rough segmentation of the region where movement is expected to occur, is available. ${ }^{10,23}$ As motion can occur outside the lungs, since the rib cage and the abdomen move during breathing, the simplest option is to use the whole patient mask. It also assumes that a prior estimation of the patient's breathing motion is available. This prior motion estimation need not be accurate: if the temporal regularization is not too strong, MA-ROOSTER will partially correct for motion misestimation.

The algorithm alternates between several optimization goals. It consists in solving the following five subproblems at each iteration of the main loop:

- Minimizing the data-attachment term, $\sum\left\|R_{\alpha} S_{\alpha} f-p_{\alpha}\right\|_{2}^{2}$, by 4D conjugate gradient (CG)

- Enforcing positivity, by setting all negative voxels of $f$ to zero

- Removing motion where it is not expected to occur, by averaging along time outside the motion mask

- Enforcing the spatial gradient's sparsity in each frame using 3D total variation (TV) denoising

- Enforcing the temporal gradient's sparsity for each spatial position, by one-dimensional (1D) TV denoising along time

In order to account for the estimated breathing motion, all frames are warped to a single reference before temporal TV denoising. The warped frames only differ where the motion estimation was inaccurate, or where the attenuation varies over time as a consequence of tissue density change (typically, the lungs are denser at end-exhale than at end-inhale). After temporal denoising, each frame is warped back to its original state.

Each supbroblem's output is used as the input for the next subproblem. This constitutes one iteration of the main loop, the output of which is fed back to the CG minimizer for the next iteration. Results are shown in figure 3. 


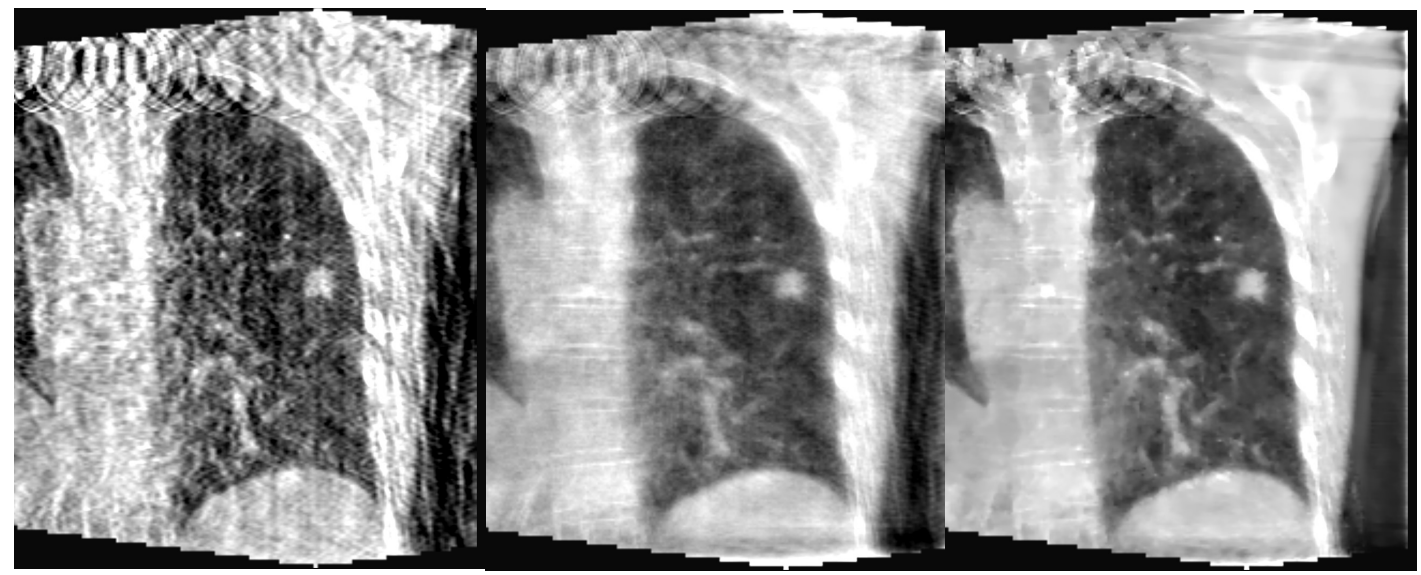

Figure 3. From left to right: 4D-FDK, 4D conjugate gradient (no regularization), MA-ROOSTER

\section{CONTRAST-ENHANCED CT}

Dynamic contrast enhanced CT can be used to measure perfusion, thus enabling a quantitative assessment of vascular integrity. Reconstruction of dynamic sequences of 3D images from complete projections acquired is unfeasible for most of small-animal cone-beam scanners because the distribution of the contrast agent changes during the acquisition and leads to inconsistent projections. We proposed a new algorithm to achieve 4D reconstructions of dynamic CT in case of slow cone-beam scanners. ${ }^{12}$ The problem of 4D reconstructions of dynamic CT in case of slow cone-beam scanners is very ill-posed as there is only one projection per time point. To tackle this we have reformulated the 4D reconstruction problem as the recovery of piecewise cubic polynomials in the temporal dimension, which was sufficient to model the input function and contrast uptake. This led to a large reduction on the number of unknowns. In order to make the problem well-posed further assumptions were assumed, such as assuming an anatomical prior is available and focusing only in those areas that change. This prior anatomical image could be obtained from an acquisition prior to contrast injection. The spatially- and temporally-dependent attenuation map, $u\left(x, y, t_{j}\right)$, was described at each time point as follows:

$$
u\left(x, y, t_{j}\right)=u_{p}(x, y)+c\left(x, y, t_{j}\right)=u_{p}(x, y)+\sum_{i=0}^{3} a_{i}(x, y) t^{i}
$$

Continuity and differentiability were imposed at the interval boundaries. The image reconstruction problem was then written as a constrained linear least-squares problem:

$$
\min _{a}\|d-G a\|_{2}^{2} \quad \text { st. } \quad A_{e q} a=b_{e q}
$$

The algorithm was assessed on a pseudo-simulated phantom consisting of four ROIs that modelled the fast input function and contrast accumulation in kidney on small animal. The proposed method yielded smooth time curves that captured the relatively fast rise and fall of contrast in the tail veil (input function), as well as the uptake and retention of contrast in the kidneys (figure 4). 

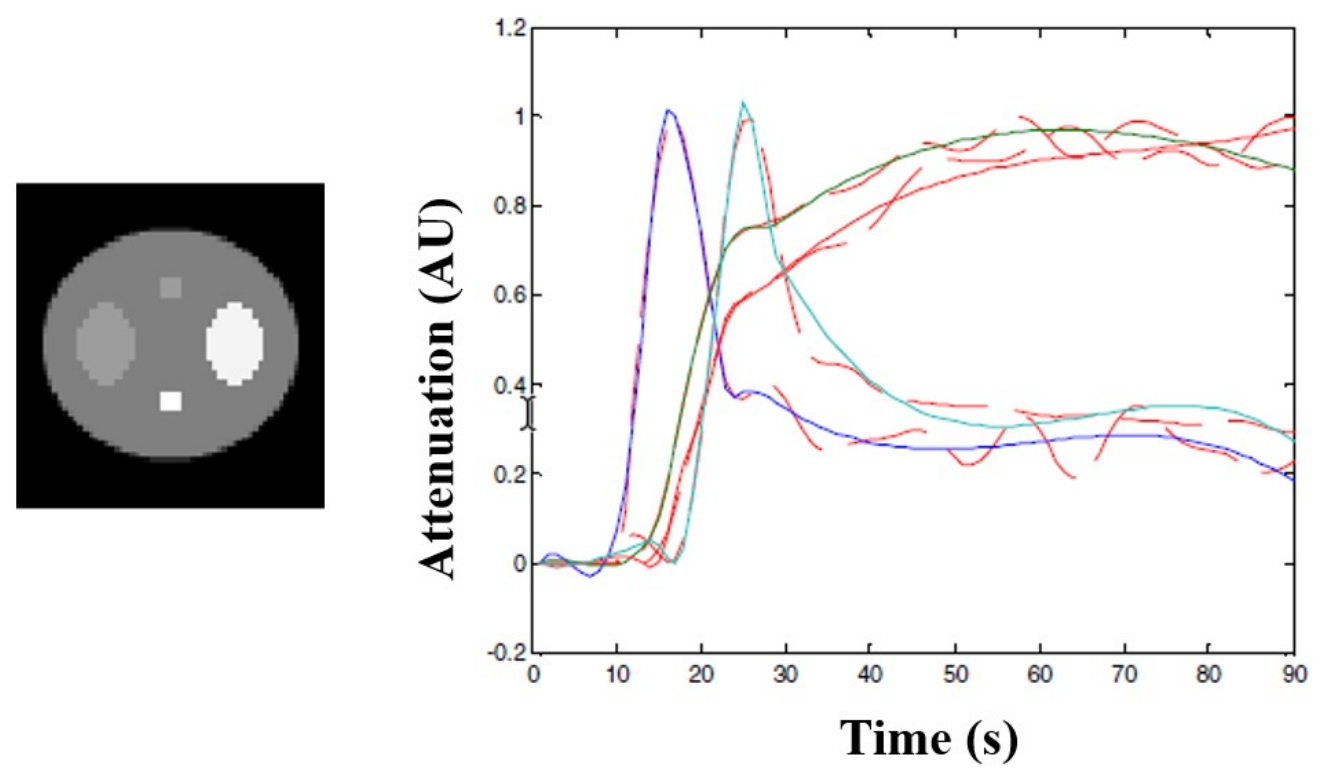

Figure 4. Fit of time curves: original curve (dotted line) and reconstructed one (continuous line) for the four ROIs.

\section{LIMITED ANGLE CT}

Limited-view CT poses the challenge of doing tomography from few projections in a limited-view angle scan. In this study we presented a preliminary study of the possibilities of solving this problem using total variation regularization based on the Split Bregman formulation. ${ }^{13,14}$ Image reconstruction was posed as the following constrained optimization problem:

$$
\min _{u}\left\|\nabla_{x, y} u\right\|_{1} \quad \text { st. } \quad\|R u-p\|_{2}^{2} \leq \sigma^{2}
$$

The method was evaluated in different simulated limited-data scenarios from a real small animal acquisition with a conebeam micro-CT scanner, considering different angular span and number of projections. Results showed that the proposed method led to a large reduction of the streak artifacts and allowed to recover the edges in the reconstructed images, compared to the FDK algorithm, in the case of angular span of 60 or 90 degrees (figure 5). 

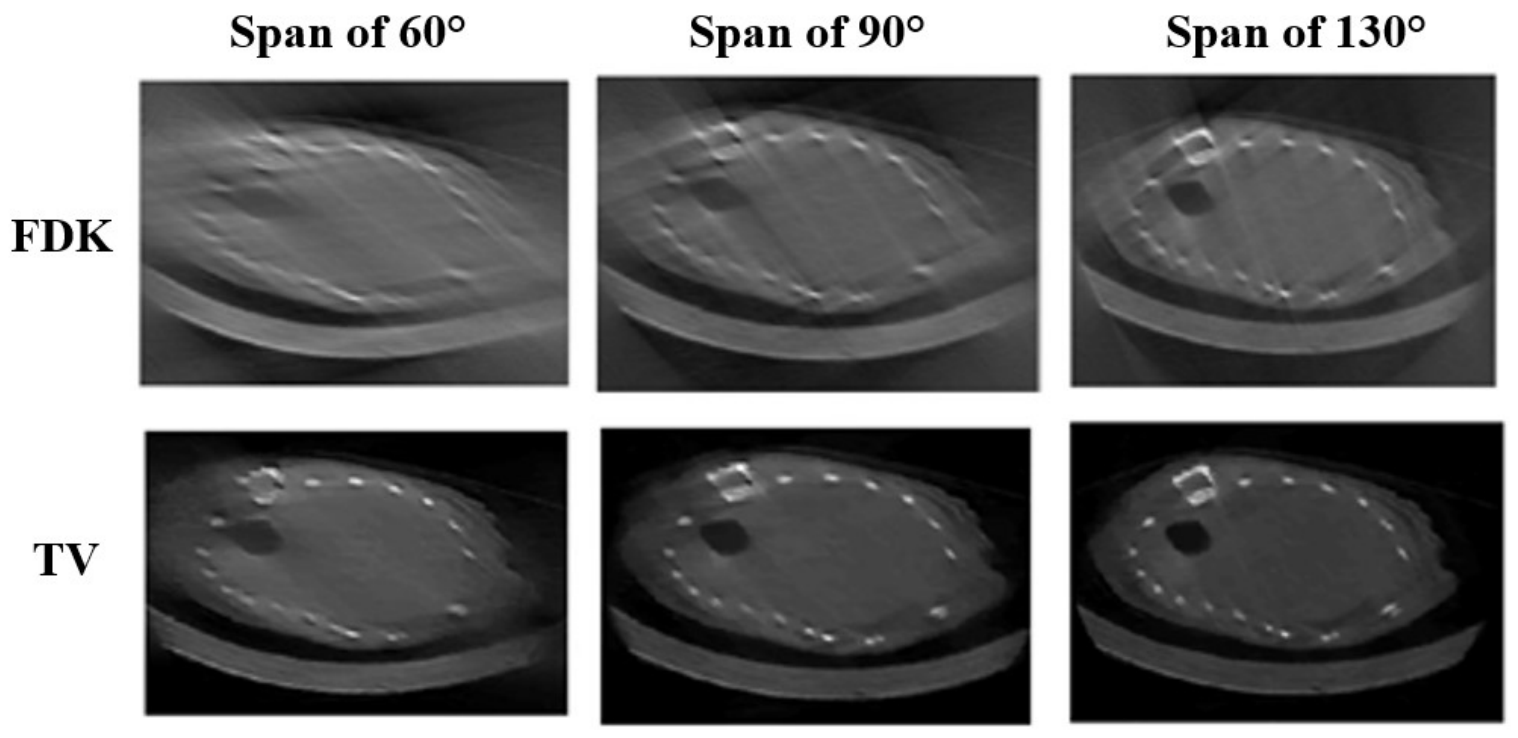

Figure 5. Reconstructed image with FDK (top) and with the proposed method (bottom). Different limited-data configurations with angular span of $60^{\circ}$ (left), $90^{\circ}$ (center) and $130^{\circ}$ (right). All with a step angle of $1^{\circ}$.

\section{SPECTRAL CT}

The new generation of Spectral Computed Tomography (SCT) scanners provide energy-dependent information that translates into material decomposition capabilities. ${ }^{24}$ However, the increase in energy resolution leads to lower signal-tonoise ratio than standard CT scanners, so new regularized reconstruction schemes are needed. The image reconstruction problem in spectral CT is commonly divided in two steps, the material decomposition problem, which is nonlinear, and the tomographic step. Mostly all studies that proposed regularized- or sparsity-based approaches focused on the tomographic step. In this study, we introduced a regularized Gauss Newton approach to exploit specific regularization for each material. ${ }^{15}$ Let $a$ be the unknown material images, $s$ the measured SCT data, $F$ the nonlinear forward operator, and $J$ a convex regularization functional. We proposed to perform material decomposition by solving the following problem:

$$
\min _{a}\|F(a)-s\|_{W}^{2}+\alpha \sum_{m} J_{m}\left(a_{m}\right)
$$

where $J_{m}$ is the specific regularization functional for the $m$-th material, $W$ is diagonal weighting matrix chosen to handle Poisson noise, and $\alpha$ is the regularization parameter. The proposed regularized weighted least squares Gauss-Newton algorithm (RWLS-GN) was assessed in a numerical thorax phantom made of up to five materials (soft tissue, bone, lung, adipose tissue, and portal vein marked with gadolinium). It was scanned with a $120-\mathrm{kV}$ source and imaged by a 4-bin photon counting detector. Data was decomposed into three materials: soft tissue, bone and gadolinium. Regularization functionals were chosen as total variation for the marker, first-order Tikhonov for bone and second-order for soft tissues. A smooth functional was assumed for soft tissue as we are working on the projection domain. The proposed method was compared to the reference maximum likelihood Nelder-Mead algorithm (ML-NM). ${ }^{25}$ MATLAB code for RWLS-GN method can be found at https://www.creatis.insa-lyon.fr/ ducros/WebPage/spray.html.

ML-NM was very sensitive to noise, leading to decomposed images highly affected by noise and artifacts. RWLS-GN was less sensitive to noise and improved contrast-to-noise ratio of the gadolinium image. In addition, the proposed method was 70 times faster than ML-NM (figure 6). 
PHANTOM

Soft tissue

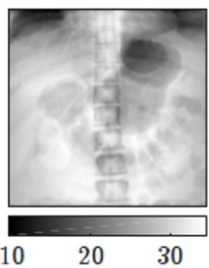

Bone

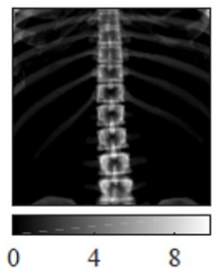

Gadolinium

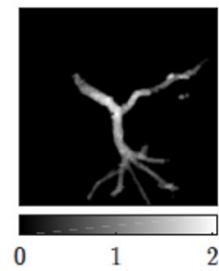

RWLS-GN

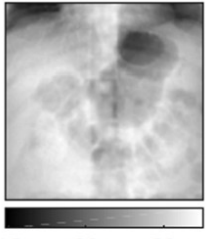

20

30

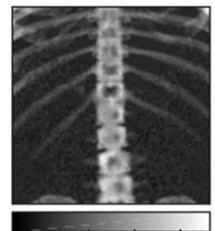

$\begin{array}{llll}0 & 2 & 4 & 6\end{array}$

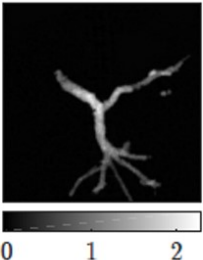

0

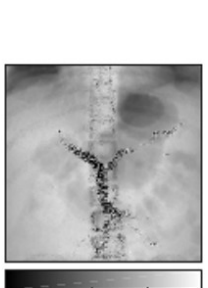

$10 \quad 20 \quad 30$
ML-NM

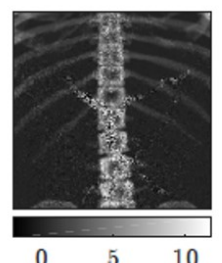

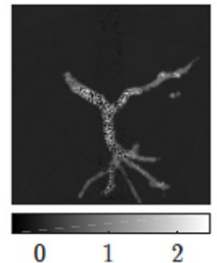

$\begin{array}{lll}0 & 1 & 2\end{array}$

Figure 6. Material decomposition of one 2D projection of a thorax phantom where the portal vein was marked with gadolinium. Top: Ground-truth material images (from left to right: soft tissue, bone, and gadolinium). Bottom: Materials imaged decomposed using the proposed RWLS-GN method (left) and the ML-NM method (right). The gadolinium concentration was set to $1 \mathrm{~g} . \mathrm{cm}^{-3}$ and the number of incident photons to $10^{7}$.

\section{DISCUSSION}

We have presented several contributions to specific X-ray CT applications where the problem is ill-posed, so regularization is needed. L1-regularization led to large improvement in image quality, with the largest benefits found for high-dimensional problems. This is the case of respiratory-gated CT, where exploiting sparsity with respect to the prior image (temporal average) allowed to significantly decrease dose and to reduce artefacts associated to respiratory movement. Further exploitation of temporal sparsity was achieved by modelling the motion between consecutive respiratory gates. This led to a significant dose reduction with respect to prior-based methods on both preclinical and clinical applications.

In enhanced contrast CT, reformulating the problem as the recovery of piecewise cubic polynomials in the temporal dimension and assuming that an anatomical prior image was available led to large reduction on the number of unknowns. This makes the problem better conditioned and has some computational advantages with respect to the common approach, as the number of unknowns is decreased considerably, but future work is required to compare the proposed methodology to more standardized approaches.

In the case of static imaging, the use of total variation regularization led to a large reduction of both streak artefacts and object distortion with respect to FDK. For this challenging problem, other functionals, such as nonlocal TV ${ }^{26}$ and generalized total variation, ${ }^{27}$ would lead to superior results than total variation.

Exploiting sparsity and generalizing compressed sensing for nonlinear problems is of current interest for many different imaging modalities. The material decomposition problem in spectral CT falls into this category, where measured projections at different energy bins are decomposed into different materials. We have shown that specific regularization for each material leads to superior results. In this case, we have combined smooth regularization functional for some materials and total variation for other material, which led to superior results. Authors believe that exploiting sparsity in spectral CT imaging has still a lot to offer.

In brief, we have shown different strategies to solve challenging problems in CT. Sparsity and more specifically L1regularization is present in most of the proposed methods. Now, we ask ourselves the following question: has sparsity reached a dead end or is the best yet to come? 


\section{ACKNOWLEDGMENTS}

This project has received funding from the European Union's Horizon 2020 research and innovation program under the Marie Sklodowska-Curie grant agreement $\mathrm{N}^{\circ}$ 701915. This work was supported by projects TEC2013-47270-R and RTC-2014-3028-1 (Spanish Ministerio de Economía y Competitividad) and DPI2016-79075-R1 (Spanish Ministerio de Economía, Industria y Competitividad). This work was performed within the framework of the LABEX PRIMES (ANR11-LABX-0063) of Université de Lyon, within the program "Investissements d'Avenir" (ANR-11-IDEX-0007) operated by the French National Research Agency (ANR). This work was also supported by the grant ANR-11-INBS-0006 of the French National Research Agency (ANR).

\section{REFERENCES}

[1] Candes, E. J.., Romberg, J. K., "Signal recovery from random projections," Proc. SPIE 5674, Comput. Imaging III, 76 , C. A. Bouman and E. L. Miller, Eds., 76, International Society for Optics and Photonics (2005).

[2] Blumensath, T., "Compressed Sensing With Nonlinear Observations and Related Nonlinear Optimization Problems,” IEEE Trans. Inf. Theory 59(6), 3466-3474 (2013).

[3] Strohmer, T., "Measure What Should be Measured: Progress and Challenges in Compressive Sensing," IEEE Signal Process. Lett. 19(12), 887-893 (2012).

[4] Song, J., Liu, Q. H., Johnson, G. A.., Badea, C. T., "Sparseness prior based iterative image reconstruction for retrospectively gated cardiac micro-CT," Med. Phys. 34(11), 4476-4483, American Association of Physicists in Medicine (2007).

[5] Pan, X., Sidky, E. Y.., Vannier, M., "Why do commercial CT scanners still employ traditional, filtered backprojection for image reconstruction?," Inverse Probl. 25(12), 1230009, NIH Public Access (2009).

[6] Persson, M., Huber, B., Karlsson, S., Liu, X., Chen, H., Xu, C., Yveborg, M., Bornefalk, H.., Danielsson, M., "Energy-resolved CT imaging with a photon-counting silicon-strip detector," Phys. Med. Biol. 59(22), 6709 (2014).

[7] Chen, G.-H., Tang, J.., Leng, S., "Prior image constrained compressed sensing (PICCS): A method to accurately reconstruct dynamic CT images from highly undersampled projection data sets," Med. Phys. 35(2), 660-663 (2008).

[8] Tang, J., Nett, B. E.., Chen, G.-H., "Performance comparison between total variation (TV)-based compressed sensing and statistical iterative reconstruction algorithms," Phys. Med. Biol. 54(19), 5781-5804 (2009).

[9] Abascal, J. F. P. J., Abella, M., Marinetto, E., Pascau, J.., Desco, M., “A novel prior- and motion-based compressed sensing method for small-animal respiratory gated CT," PLoS One 11(3) (2016).

[10] Mory, C., Janssens, G.., Rit, S., "Motion-aware temporal regularization for improved 4D cone-beam computed tomography," Phys. Med. Biol. 61(18), 6856-6877, IOP Publishing (2016).

[11] Abella García, M., Vaquero López, J. J., Sisniega, A., Reutter, B. W., Gullberg, G. T.., Desco Menéndez, M., "Statistical 4D reconstruction of dynamic CT images: preliminary results," European Society for Molecular Imaging (2009).

[12] Abella, M., Abascal, J. F. P.-J., Marinetto, E., Vaquero, J. J.., Desco, M., "Novel 4D image reconstruction for dynamic X-ray computed tomography in slow rotating scanners,” 2014 IEEE Nucl. Sci. Symp. Med. Imaging Conf. NSS/MIC 2014 (2016).

[13] De Molina, C., Abascal, J. F. P.-J., Pascau, J., Desco, M.., Abella, M., "Evaluation of the possibilities of limited angle reconstruction for the use of digital Radiography system as a tomograph," 2014 IEEE Nucl. Sci. Symp. Med. Imaging Conf. NSS/MIC 2014 (2016).

[14] de Molina, C., PJ Abascal, J., Desco, M.., Abella, M., "Study of the possibilities of Surface-Constrained Compressed Sensing (SCCS) Method for Limited-View Tomography in CBCT systems," 4th Int. Conf. Image Form. X-Ray Comput. Tomogr., Bamberg (2016).

[15] Ducros, N., Abascal, J., Sixou, B., Rit, S.., Peyrin, F., "Regularization of Nonlinear Decomposition of Spectral X-ray Projection Images."

[16] Sureshbabu, W.., Mawlawi, O., "PET/CT Imaging Artifacts*," J Nucl Med Technol 33, 156-161 (2005).

[17] Jeong, Y. J.., Lee, K. S., "Pulmonary Tuberculosis: Up-to-Date Imaging and Management," Am. J. Roentgenol. 191(3), 834-844 (2008). 
[18] Abascal, J. F. P. J., Abella, M., Sisniega, A., Vaquero, J. J., Desco, M.., Wang, G., "Investigation of Different Sparsity Transforms for the PICCS Algorithm in Small-Animal Respiratory Gated CT," PLoS One 10(4), Q. Zhang, Ed., e0120140, Kluwer Academic Publishers (2015).

[19] Goldstein, T.., Osher, S., "The Split Bregman Method for L1-Regularized Problems,” SIAM J. Imaging Sci. 2(2), 323-343, Society for Industrial and Applied Mathematics (2009).

[20] Abascal, J. F. P.-J., Chamorro-Servent, J., Aguirre, J., Arridge, S., Correia, T., Ripoll, J., Vaquero, J. J.., Desco, M., "Fluorescence diffuse optical tomography using the split Bregman method," Med. Phys. 38(11) (2011).

[21] Abascal, J. F. P. J., Abella, M., Marinetto, E., Pascau, J., Desco, M.., Ang, K., “A Novel Prior- and MotionBased Compressed Sensing Method for Small-Animal Respiratory Gated CT," PLoS One 11(3), Q. Zhang, Ed., e0149841, Public Library of Science (2016).

[22] Rueckert, D., Sonoda, L. I., Hayes, C., Hill, D. L. G., Leach, M. O.., Hawkes, D. J., "Nonrigid registration using free-form deformations: application to breast MR images," IEEE Trans. Med. Imaging 18(8), 712-721 (1999).

[23] Rit, S., Vila Oliva, M., Brousmiche, S., Labarbe, R., Sarrut, D.., Sharp, G. C., "The Reconstruction Toolkit (RTK), an open-source cone-beam CT reconstruction toolkit based on the Insight Toolkit (ITK), J J. Phys. Conf. Ser. 489(1), 12079, IOP Publishing (2014).

[24] Taguchi, K.., Iwanczyk, J. S., "Vision 20/20: Single photon counting x-ray detectors in medical imaging,” Med. Phys. 40(10), 100901, AAPM (2013).

[25] Schlomka, J. P., Roessl, E., Dorscheid, R., Dill, S., Martens, G., Istel, T., BÃoumer, C., Herrmann, C., Steadman, R., et al., "Experimental feasibility of multi-energy photon-counting K-edge imaging in pre-clinical computed tomography," Phys. Med. Biol. 53(15), 4031 (2008).

[26] Zhang, X., Burger, M., Bresson, X.., Osher, S., "Bregmanized Nonlocal Regularization for Deconvolution and Sparse Reconstruction," SIAM J. Imaging Sci. 3(3), 253-276, Society for Industrial and Applied Mathematics (2010).

[27] Bredies, K., Kunisch, K.., Pock, T., “Total Generalized Variation,” SIAM J. Imaging Sci. 3(3), 492-526, Society for Industrial and Applied Mathematics (2010). 